\title{
Perceptual Integration of Illusory and Imagined Kinesthetic Images
}

\author{
Chloé Thyrion and Jean-Pierre Roll \\ Laboratoire de Neurobiologie Humaine, Unité Mixte de Recherche 6149, Université de Provence, Centre National de la Recherche Scientifique, 13331 \\ Marseille, France
}

It is generally agreed that motor imagery involves kinesthetic sensations especially as far as first-person imagery is concerned. It was proposed to determine the extent to which motor imagery and vibration-induced illusory sensations of movement are integrated perceptually. Imagined and illusory hand movements were evoked both separately and in various combinations in 12 volunteers. After each trial, the participants were asked to draw the movement trajectory perceived. In all the subjects, propriomimetic vibration patterns applied to various wrist muscles induced spatially oriented or more complex illusory hand movements such as writing or drawing. Depending on the instructions, the subjects were also able to produce imagined hand movements in various directions and at two different velocities. When straight illusory and imagined movements were evoked simultaneously, all the subjects perceived a single movement trajectory, in which the direction and the velocity of the two ongoing sensations were exactly integrated. This perceptual integration also occurred in the case of more complex movements, such as writing and drawing, giving rise to the perception of original trajectories also combining the features of both motor images. Because these two kinesthetic images, the one intentionally and centrally induced and the other peripherally evoked, activate almost the same neural network including cortical sensory and motor areas, parietal regions, and the cerebellum, these results suggest that common processes may be involved in such a perceptual fusion. The nature of these common processes is discussed, and some fields of research in which these findings could potentially be applied are suggested.

\section{Introduction}

Both actual and first-person imagined actions give rise to conscious kinesthetic perceptions which mainly result from proprioceptive inputs or the central simulation of a sensory experience in the case of motor imagery (Annett, 1996; Naito et al., 2002; Grush, 2004; Mulder, 2007). It has also been clearly established that the neural networks underlying simulated and actually executed actions overlap considerably (for review, see Jeannerod and Frak, 1999; Lotze and Halsband, 2006), that they both elicit almost identical autonomic responses (Decety et al., 1991, 1993; Wuyam et al., 1995), and that their timing is very similar (Decety and Michel, 1989; Parsons, 1994). However, kinesthetic experiences can also be evoked by eliciting visual, cutaneous, or muscle spindle proprioceptive feedback experimentally (Goodwin et al., 1972; Roll and Vedel, 1982; Tardy-Gervet et al., 1982; Roll et al., 2002; Collins et al., 2005). The subjective perceptual content of these illusory movements is very similar to that of the kinesthetic pictures that are generated internally during imagined movements (Roll et al., 1994, 1996).

Studies focusing on muscle proprioception have shown that

\footnotetext{
Received Feb. 10, 2009; revised April 29, 2009; accepted May 23, 2009.

This research was supported by grants from Agence Nationale de la Recherche and Association Française de Lutte contre les Myopathies. We thank Dr. Jessica Blanc for revising the English manuscript.

Correspondence should be addressed to Chloé Thyrion, Laboratoire de Neurobiologie Humaine, Unité Mixte de Recherche 6149, Université de Provence, Centre National de la Recherche Scientifique, Pôle 3C, Case B, 3 Place Victor Hugo, 13331 Marseille Cedex 03, France. E-mail: chloe.thyrion@etu.univ-provence.fr.

D0l:10.1523/JNEUROSCI.0683-09.2009

Copyright $\odot 2009$ Society for Neuroscience $\quad$ 0270-6474/09/298483-10\$15.00/0
}

appropriate patterns of vibration applied to given muscle groups evoke illusory sensations of movement that can be as complex and specific as writing and drawing (Albert et al., 2006; Roll et al., 2009). These kinesthetic illusions, like kinesthetic motor images, affect the patterns of corticospinal excitability (Kito et al., 2006; Mercier et al., 2008) and, depending on the behavioral context, can evoke motor activities of cortical or reflex origin (Hagbarth and Eklund, 1966; Roll et al., 1980; Calvin-Figuière et al., 1999, 2000). Both types of kinesthetic images also activate practically the same large neural network, including sensory areas (Porro et al., 1996; Gerardin et al., 2000; Romaiguère et al., 2003; Duclos et al., 2007), cortical motor areas and the cerebellum (Naito et al., 2002), and parietal regions (Sirigu et al., 1996; Romaiguère et al., 2003). The same neural ensembles may therefore generate kinesthetic sensations in response to either the proprioceptive inputs themselves or the motor command and its associated efferent copy (Von Holst, 1954). This perceptual process can be activated internally during kinesthetic imagery and peripherally during kinesthetic illusions (Roll et al., 1994).

All these data, which suggest that common networks and neural processes may mediate kinesthetic illusions and kinesthetic imagery, recently led Kitada et al. (2002) to analyze the perceptual effects of imagined hand movements on vibration-induced illusory wrist flexion. When subjects experiencing an illusory wrist flexion movement were simultaneously imagining a hand flexion or extension movement, the maximum illusory flexion angle perceived increased or decreased, respectively.

To further investigate these processes, it was proposed to de- 
termine the extent to which illusory and imagined movements may interact perceptually. For this purpose, we combined the two perceptual experimental tasks and assessed the possible interactions by asking the subjects to actively draw the movement trajectories they perceived. In particular, we wanted to establish whether vibration-induced and imagined movements may be integrated online in a single percept that combines the direction and the velocity of the two images in the case of simple movements as well as more complex ones, such as writing or drawing.

\section{Materials and Methods}

\section{General experimental setup}

Experiments were performed on 12 healthy volunteers (three males and nine females) aged between 22 and 60 years (mean age, 36.2 years). The participants were selected because they experienced particularly vivid kinesthetic illusions in response to muscle vibration and were able to generate motor imagery. Half of these subjects were naive university students, and the others were colleagues from the laboratory who were variably familiar with the vibration procedure, but none of the 12 subjects had participated previously in motor imagery experiments. All the participants gave their informed consent to the procedure, as required by the Helsinki Declaration, and the study was approved by the local ethics committee (Comité Consultatif de Protection des Personnes dans la Recherche Biomédicele, Marseille I).

The participants were comfortably seated in an armchair with their right forearm resting on a support and their hand holding a pencil. The tip of the pencil was fixed to a digitizing tablet by means of a small suction pad. During vibration stimulation and movement imagination periods, subjects were instructed to relax, close their eyes, and focus on the perception of the movement trajectory described by the tip of the pencil. The subjects could see the experimental setup before the experiments started, and they opened their eyes after each trial.

\section{Vibratory stimuli}

Four vibrators were applied perpendicularly to the tendons of the dorsal, volar, radial, and ulnar aspects of the right wrist joint. The heads of the vibrators were specially designed to activate all the tendons of each wrist aspect (they were $1-5 \mathrm{~cm}$ long and $0.6 \mathrm{~cm}$ in diameter). The vibration was delivered via two multichannel electronic devices connected to highpower amplifiers (pulse duration, $5 \mathrm{~ms}$, regardless of the vibration frequency; amplitude, $0.25 \mathrm{~mm}$ peak-to-peak; Rematic Ltd.). The vibration frequency $(1-100 \mathrm{~Hz})$ was controlled by a software program (Labview $6 i)$.

To induce simple illusory hand movements, a vibrator was applied to the dorsal aspect of the wrist (illusory hand flexion movements), the volar aspect (illusory hand extension movements), or the radial aspect (illusory hand adduction movements), and the vibration frequency was held at 80 or $35 \mathrm{~Hz}$ for $7 \mathrm{~s}$. More complex illusory movements (an alternating abduction-adduction movement, period of $2 \mathrm{~s}$; the drawing of a square, duration of $8 \mathrm{~s}$; and the writing of the letter $\mathrm{m}$, duration of $6 \mathrm{~s}$ ) were induced by applying vibration patterns generated by a "proprioceptive model" exactly mimicking the proprioceptive afferent feedback evoked by the corresponding actual movements.

This proprioceptive model was described in detail in a recent study (Roll et al., 2009). Briefly, it is based on the movement coding properties of populations of muscle spindle afferents recorded with microelectrodes inserted into the peripheral nerves of human subjects (the microneurographic method). Microneurographic recordings obtained at leg and arm levels under active and passive conditions brought to light the following properties of single muscle afferents and whole afferent populations. Each muscle spindle responds only to a specific range of movement directions (corresponding to the "preferred sensory sector") and shows maximum sensitivity in a specific direction (the "preferred sensory direction"). When moving away from this direction, the activity of the receptor is decreasingly cosine tuned. The mean activity of a muscle spindle population during a movement trajectory is expressed in terms of a "population vector." The direction of this population vector is the preferred sensory direction of a given muscle, whereas its module is proportional to the mean firing rate of the muscle spindle population.
Based on the total sensory activity originating from all the muscles acting on a given joint (i.e., by summing all the population vectors), it was established that the neurosensory sum vectors are highly correlated with the movement vectors (the tangential velocity vectors) at any point on a given trajectory. Each sum vector therefore gives the instantaneous direction and velocity of the ongoing movement.

Based on these neurophysiological properties of proprioceptive receptors, our model consists of running the process backwards, i.e., starting with the movement vectors calculating the underlying population vectors corresponding to the oriented and weighted contribution of each receptor population to the instantaneous coding of the ongoing movement. Each sum vector is then projected orthogonally onto the preferred sensory direction of each muscle to simulate the cosine tuning of the muscle spindles, which restricts the preferred sensory sector to $180^{\circ}$.

To generate the simulated afferent patterns, all one therefore needs to feed into the model are the movement trajectory and the preferred sensory directions of the main muscle groups activating a given limb segment. These modeled afferent patterns, which are highly correlated with the actual patterns recorded, can then be used as vibratory patterns to evoke the corresponding illusory movements.

\section{Motor imagery}

Training session. Before the experimental session, a training session was run. Before the subjects were asked to produce imagined movements, vibratory patterns were applied to their wrist muscles to induce the corresponding illusory movements. Because previous motor execution enhances the vividness of motor imagery (Jeannerod, 1994), the subjects were then instructed to draw the trajectory they had just perceived on the tablet by means of voluntary movement, paying special attention to the direction and the velocity. Last, the subjects were asked to mimic the illusory movement by imagining themselves performing it. Each task was performed three times, and the entire procedure was repeated in the case of three simple hand movements (flexion, extension, and adduction, duration of $7 \mathrm{~s}$ ) and two more complex hand movements (an alternating flexion-extension movement, period of $2 \mathrm{~s}$; and the drawing of a square, duration of $8 \mathrm{~s}$ ). Last, the subjects were trained to imagine simple wrist flexion or extension movements performed as slowly as possible.

The timing of the imagined movements was controlled by delivering beeps to the subjects informing them when to start and stop imagining the movements or to make changes of movement direction. The timing patterns imposed corresponded exactly to those of the various illusory movements.

EMG monitoring. During the training session, the ability of the subjects to imagine movements without actually performing any significant motor activity was checked by making EMG recordings. Surface EMGs (2 $\mathrm{cm}$ between leads; Contrôle Graphique Médical) were recorded from the extensor carpi radialis longus and brevis muscles (ECR) (representing the wrist extensor muscles) and the flexor carpi radialis muscle (FCR) (representing the wrist flexor muscles). An audio monitor (Grass Instruments) was used to deliver auditory feedback about the EMG signals to the subjects so that they could gradually learn to avoid making any associated muscle contractions during the kinesthetic imagery task. The procedure was repeated until the auditory feedback delivered during the motor imagery task become null, and the number of trials required therefore varied from one subject to another.

A few days later, subjects underwent an EMG recording session to quantify the motor activity possibly associated with the imagined movements. Surface EMGs were recorded from the ECR and FCR during the imagined performance of four simple movements (hand flexion, hand extension, slow hand flexion, and slow hand extension) in the absence of auditory feedback, as well as during maximum voluntary contraction (MVC) of the two muscle groups. The signals recorded were processed offline using the Spike 2 software program (Cambridge Electronic Design). After filtering and background noise removal, rectified and integrated EMGs were computed from the last $3 \mathrm{~s}$ of each imagined movement and during $3 \mathrm{~s}$ of MVC executed under isometric conditions. The 3 s rectified integrated EMGs were then expressed as a percentage of the $\mathrm{MVC}$ in the case of each recording. Figure 1 illustrates the EMG activity recorded during the training and the posttraining sessions in two differ- 
1

A

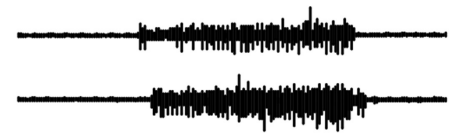

B

C

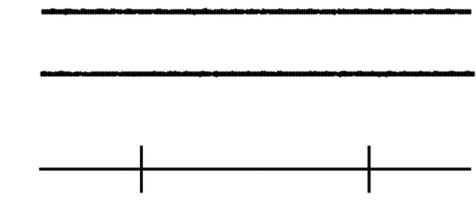

2
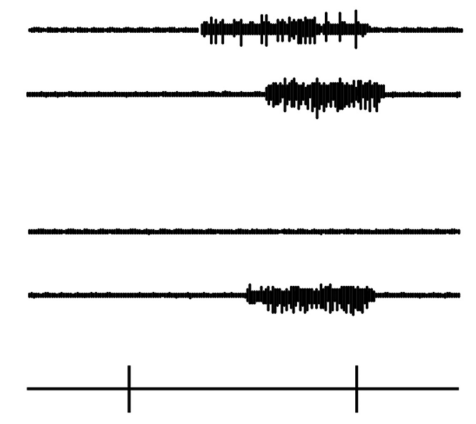

3

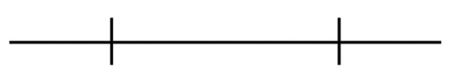

$0.25 \mathrm{mV}$

$5 \mathrm{sec}$

Figure 1. EMG correlates of imagined hand extension movements. Surface EMG recordings from the extensor carpi radialis obtained during the training session $(\boldsymbol{A})$ and the posttraining session (B) in two different subjects (top and bottom traces). On each line, three successive EMG recordings obtained during the training session and the posttraining session are presented. C, Beeps delivered to the subjects informing them when to start and stop imagining the hand extension movement.

Table 1. Rectified and integrated EMG activities recorded during simple imagined movements

\begin{tabular}{lllll}
\hline Subjects & $\mathrm{IM}(\uparrow)$ & $\mathrm{IM}(\downarrow)$ & $\mathrm{IM}(\uparrow)$ slow & $\mathrm{IM}(\downarrow)$ slow \\
\hline 1 & 2.74 & 1.39 & 2.95 & 1.75 \\
2 & 2.04 & 0.07 & 2.32 & 0.00 \\
3 & 0.36 & 0.01 & 0.38 & 0.00 \\
4 & 0.96 & 0.27 & 2.56 & 0.54 \\
5 & 0.03 & 0.03 & 0.01 & 0.03 \\
6 & 0.63 & 2.14 & 3.31 & 0.22 \\
7 & 0.69 & 0.04 & 1.18 & 0.03 \\
8 & 0.96 & 0.00 & 1.41 & 0.04 \\
9 & 0.20 & 0.03 & 0.00 & 0.00 \\
10 & 0.02 & 0.02 & 0.10 & 0.01 \\
11 & 0.00 & 0.00 & 0.00 & 0.00 \\
12 & 0.98 & 2.65 & 1.13 & 0.34 \\
Mean & 0.80 & 0.55 & 1.28 & 0.25 \\
SDs & 0.84 & 0.91 & 1.18 & 0.48 \\
\hline
\end{tabular}

From left to right, each column corresponds to an imagined movement in a given direction with two differen velocities [hand extension, IM ( $\uparrow$ ); hand flexion, $\mathrm{IM}(\downarrow)$; slow hand extension, $\mathrm{IM}(\uparrow)$ slow; and slow hand flexion, IM ( $\downarrow$ ) slow]. Lines 1-12 give the individual rectified and integrated EMG activities expressed as a percentage of each subject's MVC. The last two lines give the mean EMG values and the corresponding SDs.

ent subjects. All of the subjects were able to imagine hand movements without generating any significant associated EMG activity (mean percentage, $<1 \%$; maximum, $3.3 \%$ ) (Table 1 ).

\section{Experimental conditions}

The first set of experiments focused on the perceptual interactions between simple illusory and simple imagined hand movements. Illusory hand adduction movements were induced by applying vibration to the radial aspect of the right wrist joint. The velocity of these illusory movements depended on the vibration frequency ( 80 or $35 \mathrm{~Hz}$ ) (Roll and Vedel, 1982; Jones, 1988). The imagined movements were hand flexion or extension movements executed at two different velocities (either at approximately the same velocity as the $80 \mathrm{~Hz}$ illusory movement or as slowly as possible). Imagined and illusory movements were first evoked independently and then combined in different ways. Figure 2 shows the experimental setup, along with the various combinations of simple imagined and illusory movements (Fig. 2 B).

The second set of experiments focused on the perceptual interactions between more complex illusory and imagined movements, such as writing and drawing movements. The vibration-induced illusory movements involved drawing the letter $\mathrm{m}$, the geometrical figure square, adduction, and alternating abduction-adduction hand movements. The imagined movements were simple hand extension or adduction movements, alternate flexion-extension movements, and those required to draw more complex geometrical figures such as a square. Illusory and imagined movements were first evoked separately and then combined in different ways (Fig. 2C).

In both sets of experiments, the subjects were informed about the category of movement liable to be perceived, i.e., simple movements, letters or geometrical shapes, and each sequence was repeated three times. Then, whether the movements were illusory or imagined and evoked separately or combined, their parameters were quantified by asking the subjects to draw the trajectory perceived on a digitizing tablet, paying special attention to the direction and velocity in the first set of experiments and to the shape, size, and velocity in the second set. The movement trajectory drawn by each participant was then sampled at 200 $\mathrm{Hz}$ and stored for additional analysis. Last, with the last two combinations of complex imagined and illusory movements, the subjects were also asked to identify the trajectories they perceived. All in all, the whole experiment was composed of 23 different items, and each item was repeated three times.

\section{Data analysis}

The various trajectories drawn to depict the simple imagined and illusory movements evoked either separately or combined were repositioned with a common starting point and expressed by vectors. First, the straightness of the trajectories was rated using an index defined as the ratio between the straight distance from the starting point to the endpoint (D) and the actual length of the trajectory (L) (Benhamou, 2004). Because the values of all the $\mathrm{D} / \mathrm{L}$ indices were approximately equal to 1 (mean $\pm \mathrm{SD}, 0.9 \pm 0.06$; minimum, 0.74 ), linear regression equations were computed with all the trajectories, which were then replaced by the corresponding straight regression lines to determine their orientation. The mean velocity was calculated after removing the initial and final parts of the trajectory, during which the velocity was not constant (using the velocity profiles). Each trajectory was thus modeled by a vector pointing in the movement direction, the norm of which was proportional to the mean velocity.

The velocities of separately evoked simple movements were first compared to check whether the vibration frequency affected the perceived velocity of the illusory movements and whether the instructions affected the perceived velocity of the imagined movements. Paired $t$ tests or Wilcoxon's tests were used for this purpose, depending on the normality of the distributions (which was determined using the Shapiro-Wilks test). Circular statistical analyses (Batschelet, 1981) were then performed. The $V$ test was first applied to the vectors corresponding to separately evoked illusory and imagined movements to check whether clusters occurred 
around the expected orientation, depending on the vibratory patterns or the instructions given to the subjects.

To determine the extent to which vibrationinduced and imagined movements are integrated in terms of their direction and velocity, a theoretical vector was then obtained for each subject in each trial by summing the vectors of separately evoked imagined and illusory movements. The $V$ test was also used here to test whether the experimental vectors corresponding to combined imagery and illusion tasks tended to point in the direction of the corresponding theoretical vectors. For this purpose, each subject's angular deviation was calculated with respect to the direction defined by the corresponding theoretical vector, and the $V$ test was performed to determine whether these deviations were uniformly distributed in a circle or clustered around $0^{\circ}$. The Watson-Williams test was also used to compare the experimental vectors obtained under the various experimental conditions.

In the second set of experiments, various parameters were used to examine the interactions between more complex imagined and illusory movements. In the task involving the drawing of squares, the areas of the squares, and the velocities at which they were traced were compared between the three experimental conditions. Because the areas were not normally distributed (Shapiro-Wilks test), a nonparametric test (Friedman's test) was used, combined with the Wilcoxon's test for pairwise comparisons. Because the mean velocities of the trajectories drawn were normally distributed, the ANOVA for paired data and the NewmanKeuls test for pairwise comparisons were used here. In the task in which the letter $\mathrm{m}$ was written, each letter drawn by the subjects was placed in a rectangle to compare the width $(x)$ and height $(y)$ of the letters traced under the various conditions and to determine the extent to which the trajectories were distorted when illusory and imagined movements occurred concomitantly. Depending on the normality of the distributions, the Wilcoxon's test or the paired $t$ test was performed.

Combining an imagined alternating flexionextension movement with an illusory movement to the right was expected to result in the perception of a sinusoidal movement, whereas the same imagined sequence combined with an illusory alternating abduction-adduction movement shifted by a half-period was expected to result in the perception of a circle-like trajectory. One of the criteria used to determine whether any interactions occurred between the two motor images was whether or not the subjects identified the perceived trajectories correctly. Then, the average shapes of all the trajectories drawn by the subjects on the digitizing tablet (based on the mean $x$ and $y$ coordinates) were computed after lining up the individual trajectories with a reference point corresponding to the end of the first movement period. From the mean sinusoid obtained, a theoretical sinusoid was computed by relating the various parameters using the following sine function: $y=a^{*} \sin$ $((2 p / p) * x+c)$, where $a$ is the amplitude, $p$ is the period, and $c$ is the phase. In the same way, a theoretical circle was determined from the mean radius of the average circle obtained. Because the $x$ and $y$ coordinates of the trajectories were not normally distributed ( $\chi^{2}$ test), two nonparametric tests were used to compare the theoretical and average hand-drawn shapes (Spearman's correlation and Mann-Whitney test).

Last, the average shape of each trajectory drawn on the digitizing tablet in both sets of experiments was determined from all the trajectories so that visual comparisons could be made.

\section{Results}

In all the subjects, wrist muscle vibration applied at a constant frequency evoked an illusory hand movement, the direction of which was that of the movement that would have stretched the vibrated muscle group. The movement velocity perceived depending on the vibration frequency used ( 35 or $80 \mathrm{~Hz}$ ) was almost constant. The illusory sensation of movement disappeared as soon as the vibration stopped. In some subjects, kinesthetic post-effects could occur in the opposite direction to that of the previous illusion. After motor imagery training, all the subjects were able to imagine hand movements without producing any significant associated EMG activity (see Materials and Methods) (Table 1).

When kinesthetic pictures were jointly evoked by muscle tendon vibration and motor imagery, it is worth mentioning that the subjects never felt two separate kinesthetic sensations but a single 
A
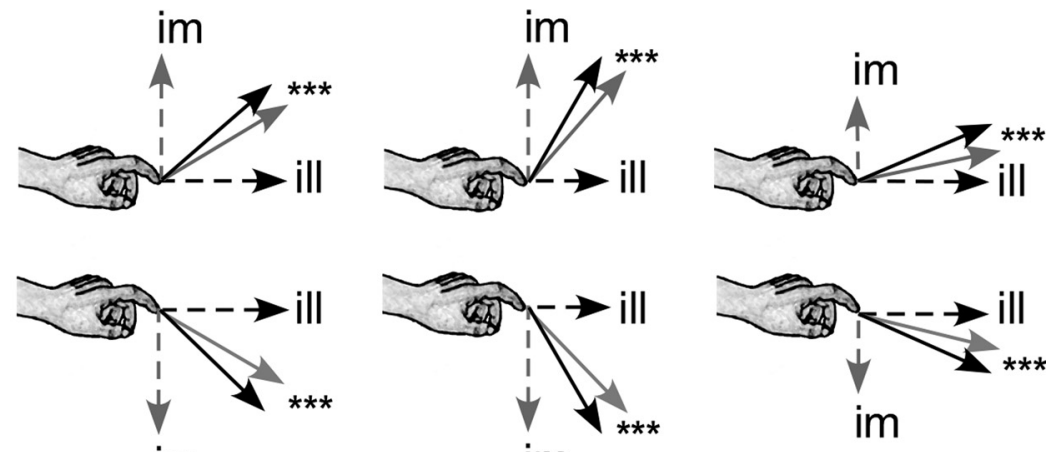

im
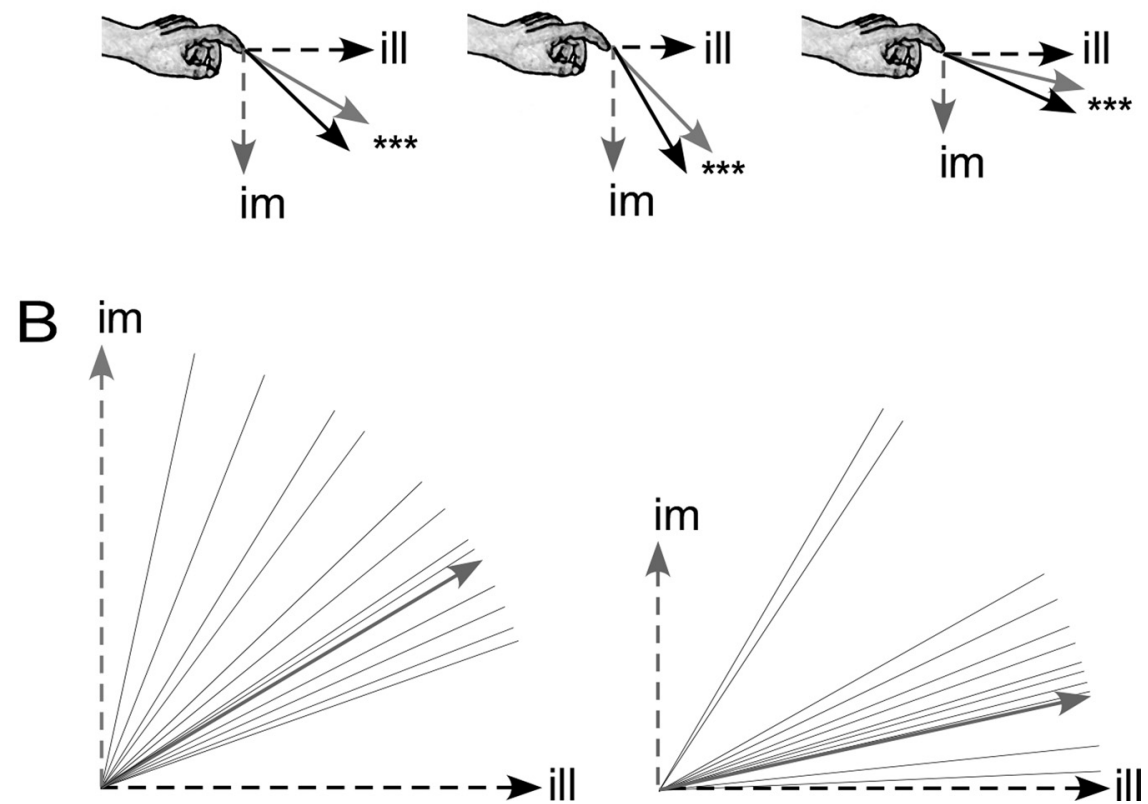

Figure 3. Perceptual integration of simple illusory and imagined movements. $\boldsymbol{A}$, Mean experimental results ( $n=12$ subjects) obtained in the various conditions tested in the first set of experiments. Imagined (im) and illusory (ill) movement vector lengths are proportional to either the vibration frequency used ( $80 \mathrm{~Hz}$, dashed long black arrow; or $35 \mathrm{~Hz}$, dashed short black arrow) or the imagined movement velocity (fast, dashed long gray arrow; or slow, dashed short gray arrow). In all the experimental conditions tested, the mean experimental vectors (dark arrows) were in the close vicinity of the theoretical vectors (gray arrows), which indicates that perfect perceptual integration of the two simultaneously evoked kinesthetic sensations occurred, in terms of their orientation and velocity. ${ }^{* * *} p<0.001, V$ test. $\boldsymbol{B}$, Variability of the findings across subjects in two of the six experimental conditions studied. The same conventions as in $\boldsymbol{A}$ were used here to present illusory (ill) and imagined (im) movement vectors, as well as mean theoretical vectors. The individual data (thin gray lines) are represented as the angular distance from the mean theoretical vector.

one, the perceptual characteristics of which corresponded to a combination of the two kinds of motor images.

\section{Simultaneously induced illusory and imagined movements gave a combined picture in terms of direction and velocity} At both frequencies tested ( 80 and $35 \mathrm{~Hz}$ ), vibration applied to the wrist abductors evoked an illusory hand adduction movement, i.e., an illusory horizontal movement to the right, in all the subjects. Likewise, in the kinesthetic imagery tests, all the subjects were able to imagine a vertical upward or downward hand movement as instructed. When expressed in the form of vectors, the directions of the illusory trajectories clustered around $90^{\circ}$ ( $V$ test, $v=0.996 ; u=6.902 ; p<0.001)$, and the imagined upward trajectories clustered around $0^{\circ}$ ( $V$ test, $v=0.996 ; u=6.902 ; p<$ $0.001)$, whereas imagined downward trajectories clustered around $180^{\circ}$ ( $V$ test, $v=0.994 ; u=6.884 ; p<0.001$ ). The perceived directions of the illusory and imagined trajectories were therefore orthogonal.

The mean velocity of the illusory movements given by the vector lengths was $25.8 \mathrm{~mm} / \mathrm{s}$ when the vibration frequency was set at $80 \mathrm{~Hz}$ and $12.6 \mathrm{~mm} / \mathrm{s}$ when the vibration frequency was set at $35 \mathrm{~Hz}$. These two velocities were significantly different (paired $t$ test, $t=5.157 ; p<0.001$ ). In the case of imagined movements, the mean velocity of the simple movements mimicking the $80 \mathrm{~Hz}$ illusion was $15.1 \mathrm{~mm} / \mathrm{s}$, whereas the mean velocity of those performed "as slowly as possible" was $6 \mathrm{~mm} / \mathrm{s}$. These two velocities also differed significantly (Wilcoxon's test, $p<0.01$ with both upward and downward imagined movements).

When illusory and imagined simple movements were combined, all the subjects perceived and then drew a single oblique trajectory, the direction of which was midway between the two trajectories evoked separately.

The mean theoretical vectors giving the resulting perceptions expected to occur if the two motor images were fully integrated are shown in Figure 3, along with the mean experimental vectors computed from the trajectories perceived and copied by all the subjects under each of the six experimental conditions. Results showed that the mean experimental vectors were very similar to the theoretical ones: the fact that the directions of the resulting perceptions showed a significant tendency ( $V$ test, $v=0.919 ; u=11.029 ; p<0.001)$ to cluster around the directions of the theoretical vectors indicates that both the velocity and the direction of the two sensations were taken into account in the combined perception. In addition, a significant difference was observed between the orientations of the mean experimental vectors obtained under the various conditions (Watson-Williams test, $p<0.001$ ) (Table 2).

Jointly evoked vibration-induced and imagined movements are therefore integrated perceptually and result in a single perception that exactly combines the direction and velocity of the two corresponding kinesthetic images.

\section{Spatiotemporal integration of illusory and imagined writing and drawing movements}

When more complex vibration patterns were applied to the wrist muscles, all the subjects had the clear impression that their hand was drawing a square or writing the letter $\mathrm{m}$.

In the first three experimental conditions, whether the perceived trajectories were vibration-induced, imagined, or resulted from the simultaneous perception of both kinds of images, the resulting perception was that of drawing a square. Figure $4 \mathrm{~A}$ shows the individual and mean squares obtained under the three conditions. The areas and the perceived velocity of the vibrationinduced and imagined mean squares were both significantly smaller than those observed with the simultaneously induced square (Friedman's test, $F_{\text {areas }}=20.67 ; p<0.01$, Wilcoxon's test, $p<0.05$ for the two pairwise comparisons; ANOVA for paired data, $F_{\text {velocities }}=21.08 ; p<0.00001$, Newman-Keuls test, $p<$ 0.01 for the two pairwise comparisons) (Table 3).

Another vibratory pattern evoked the illusory sensation that the hand was writing the letter $\mathrm{m}$. This is illustrated in Figure $4 B$, which gives the mean shapes perceived under the three experimental conditions. When simple imagined movements were 
added while this illusion was being evoked, subjects still perceived the letter $\mathrm{m}$, although they reported that the writing trajectory was distorted and/or reoriented in specific ways. Imagined hand movements toward the right caused distortion of the letter $\mathrm{m}$ along the $x$-axis (paired $t$ test, $t=4.213 ; p<0.0015)$ but not along the $y$-axis (Wilcoxon's test, nonsignificant), whereas imagined upward hand movements caused distortion of the letter $\mathrm{m}$ along the $y$-axis (Wilcoxon's test, $p<$ 0.01 ) but not along the $x$-axis (paired $t$ test, nonsignificant).

\section{Combining some illusory and imagined movement patterns gave rise to original motor shapes}

When imagined alternating flexion-extension hand movements were combined with an illusory movement to the right, all the subjects reported that the tip of the pencil clearly described a sinusoid. The average sinusoid (Fig. 4C) computed from all the trajectories drawn did not differ significantly from the theoretical sinusoid (the Mann-Whitney test on the $x$ and $y$ coordinates was nonsignificant). Moreover, the expected and experimental shapes were highly correlated (Spearman's correlation, $r_{x}=1, r_{y}=0.877$ ).

When imagined alternating flexion-extension hand movements were combined with a vibration-induced illusion of alternating abduction-adduction, the great majority of the subjects ( 9 of 12) clearly perceived that the tip of the pencil described a circle, whereas the others reported a lozengeshaped trajectory with rounded angles. The average shape (Fig. 4C) computed from all the trajectories drawn did not differ significantly from a theoretical circle (the Mann-Whitney test on the $x$ and $y$ coordinates was nonsignificant), and the two trajectories were highly correlated (Spearman's correlation, $\left.r_{x}=0.991, r_{y}=0.999\right)$.

All in all, the present results show that simple vibrationinduced illusory movements and simultaneously imagined movements are fused together in terms of their orientation and velocity. When more complex illusory and imagined movements were induced simultaneously, they gave rise to a single original percept combining the spatial and temporal parameters of the two kinesthetic images evoked separately.

\section{Discussion}

In all the subjects, propriomimetic vibration patterns applied to various wrist muscles induced spatially oriented hand movements or more complex illusory hand movements, such as writing or drawing. When a simple illusory movement was combined with an imagined orthogonal movement, all the subjects perceived a single movement trajectory that integrated the direction and the velocity of the two ongoing kinesthetic sensations. This perceptual integration also occurred in the case of more complex movements such as writing or drawing, giving rise to the perception of original trajectories also combining the spatiotemporal features of both motor images.

\section{What do the subjects feel? Subjective reports}

What exactly do subjects perceive when they are receiving vibration around the wrist, when they are imagining the same movement, and when illusory and imagined movements are combined?

The vibration-induced illusory movements were clearly perceived by all the subjects as being hand movements with a given direction and velocity. In most cases, the subjects were convinced that the illusory movement had actually occurred. Based on subjects' responses to a questionnaire (Roll et al., 1996), it emerged that the sensation of movement was perceived as resulting from "external" forces by only $25 \%$ of the subjects and as resulting from "internal" (although nonintentional) forces by the remaining $75 \%$. Seventy-five percent of the subjects experienced a feeling of strangeness, probably because they seemed to be performing an action without their own volition but possibly also because the proprioceptive sensory feedback triggered by the vibratory stimulus elicited only part of the "sensorimotor landscape" that normally surrounds a voluntary action. Here no muscles were voluntarily activated and no feedback usually arising from the Golgi tendon organs was evoked. In addition, because no motor command was involved, no "efferent copy" was generated such as that which partly mediates the conscious awareness of real movements (Teuber, 1972; Jaeger et al., 1979; McCloskey, 1981; Smith et al., 2009). These lacking components might therefore explain why the kinesthetic illusions induced by vibration are qualitatively slightly incomplete. The apparently somewhat contradictory conclusion that can be drawn from the above findings is that the illusory sensations of movements were in fact very similar to those that normally accompany voluntary actions, although the subjects did not generate them intentionally.

Subjective reports about the kinesthetic sensations associated with imagined movements are better known from the 
A
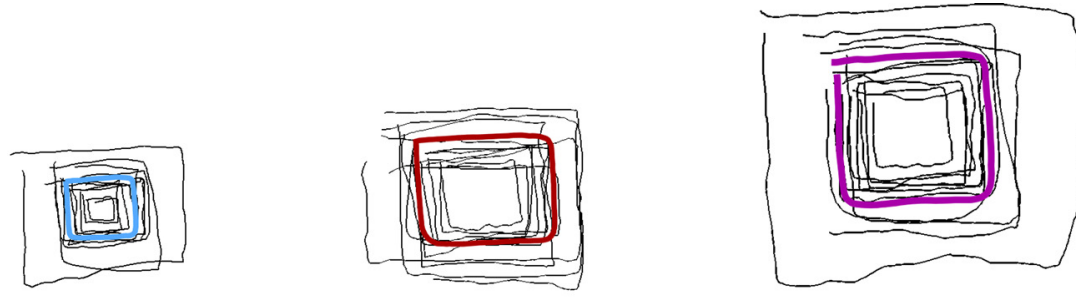

B
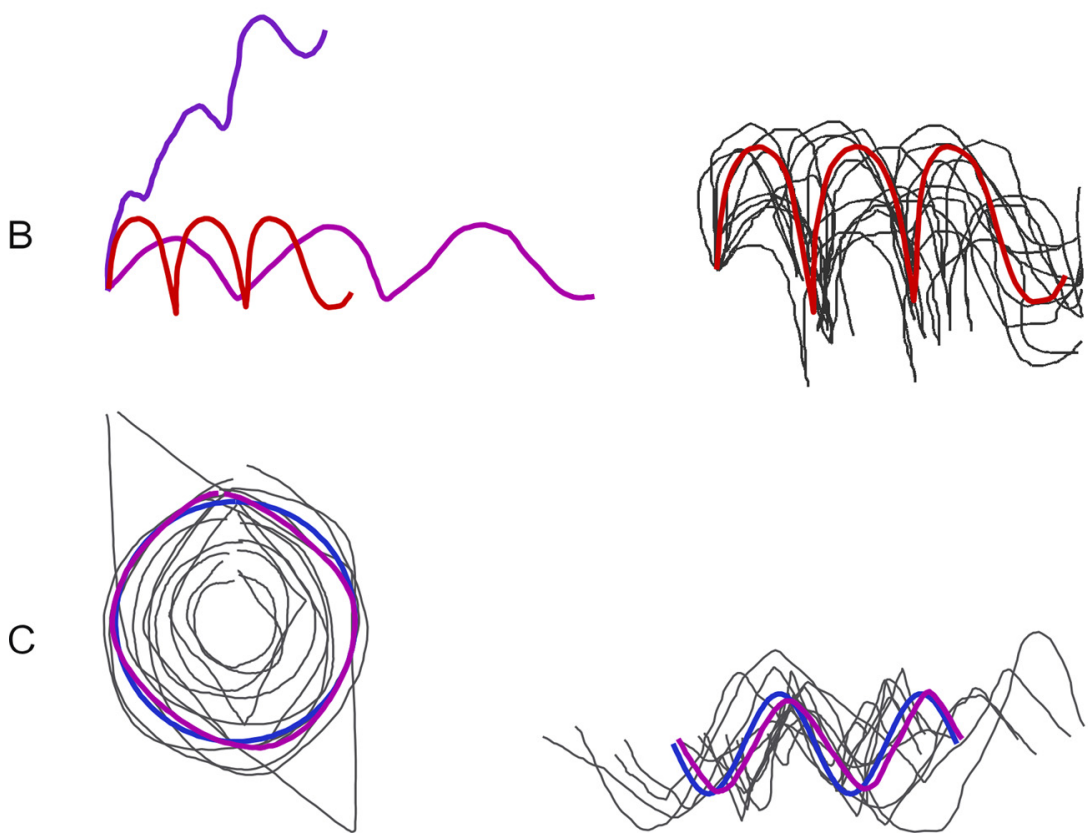

Figure 4. Perceptual integration of complex illusory and imagined movements. $\boldsymbol{A}$, Mean square trajectories computed with all the subjects: mean vibration-induced illusory square (red trace), mean imagined square (blue trace), and mean combined square (purple trace). All the subjects' drawings (thin black lines) are superimposed on the mean trajectories obtained in each experimental condition. $\boldsymbol{B}$, Mean letter $\mathrm{m}$ trajectories computed with all the subjects: mean vibration-induced illusory letter $\mathrm{m}$ (red trace); mean perceived letter $m$ trajectory when an imagined movement to the right and an illusory letter $m$ were evoked simultaneously (pale purple trace); and mean letter $m$ trajectory perceived when an upward imagined movement and an illusory letter $\mathrm{m}$ were evoked simultaneously (purple trace). All the subjects' drawings (thin black lines) are superimposed on the mean vibration-induced letter $m$ trajectory. C, Thin gray traces, Individual subject's drawings of an imagined alternating flexionextension movement combined with an illusory alternating abduction-adduction movement shifted by a half-period (left) or with an illusory movement to the right (right). Purple traces, Mean circular and sinusoidal trajectories. Blue traces, Expected circular and sinusoidal trajectories.

Table 3. Illusory and imagined drawing movements (a square): numerical data

\begin{tabular}{|c|c|c|c|c|}
\hline & Squares area $\left(\mathrm{cm}^{2}\right)$ & & Squares velocity $(\mathrm{cm} / \mathrm{s})$ & \\
\hline \multicolumn{5}{|l|}{$A$} \\
\hline Illusory square & 14.81 & & 1.86 & \\
\hline Imagined square & 10.38 & & 1.61 & \\
\hline Combined square & 30.67 & & 2.56 & \\
\hline \multicolumn{5}{|l|}{ B } \\
\hline$F$ & 20.67 & & 21.08 & \\
\hline$p$ & $<0.01$ & & $<0.00001$ & \\
\hline Comparisons & IM vs combined & ILL vs combined & IM vs combined & ILL vs combined \\
\hline$p$ & $<0.05$ & $<0.05$ & $<0.01$ & $<0.01$ \\
\hline
\end{tabular}

$A$, Left column, Mean areas of illusory, imagined, and combined squares. Right column, Mean velocities at which illusory, imagined, and combined squares were drawn by hand. $B$, Top, Corresponding statistical comparisons (Friedman's test on square areas and ANOVA for paired data on square velocities) and associated $p$ values $(p)$. Bottom, $p$ values associated with the pairwise comparisons (Wilcoxon's test on square areas and Newman-Keuls test on square velocities) between imagined (IM), illusory (ILL), and combined squares.

literature (Annett, 1995; Mulder, 2007). What constantly emerges is the fact that these sensations clearly seem to be intentional and involve a sense of ownership. This intentional component therefore seems to be the only really noticeable difference between the two kinds of kinesthetic sensations despite the fact they both involve a definite sense of ownership.

Last, when illusory and imagined movements were combined, the subject's answers to questions about the qualitative aspects of the resulting perceptions all agreed as to their uniqueness. In addition, because the subject's attention was focused on the parameters of the ongoing trajectory, the intentional component associated with imagined movements was obviously toned down (Rosenkranz and Rothwell, 2004, 2006): the subjects were no longer clearly aware of their intentionality and perceived a single motion picture that was quite different from that they were continuing to imagine. Indeed, surprisingly, all the subjects reported that they continued to generate the imagined movement, although the perceived trajectory of the movement changed.

Instantaneous spatiotemporal integration of kinesthetic illusions and kinesthetic imagery

The movement parameters perceived in the case of kinesthetic imagery are determined by the task requirements and the subjects' ability to produce the imagery requested, whereas in the case of kinesthetic illusions, the movement trajectory and its velocity are determined by the vibration pattern applied (Roll and Vedel, 1982; Albert et al., 2006; Roll et al., 2009).

In the present study, the spatial and temporal parameters of each separately evoked kinesthetic sensation were calculated to predict each subject's perception of the combined illusory and imagined movements. The data obtained show that the resulting perception corresponded exactly to the sum of the vectors giving the velocity and direction of each of the two sensations of movement. It therefore seems likely that both kinesthetic images contributed in equal proportions to the resulting perception. Thus, the present findings may suggest that these two kinds of motor images are reciprocally integrated. Interestingly, when the direction of the illusion and that of the imagined movement were similar, the movement velocity perceived was faster and the subjective perception was more vivid.

The results obtained here on the perception of complex illusory and imagined movements show that the spatiotemporal parameters characterizing each of the two separately evoked kinesthetic images are exactly integrated when these 
sensations are induced simultaneously. In particular, the fact that the subjects perceived a sinusoid or a circle although each kinesthetic image included only some of the corresponding constitutive spatiotemporal parameters shows that a perfect ongoing perceptual integration of the two images took place. In addition, the resulting perception could be predicted mathematically, because two sinusoids shifted by a half-period give a circular pattern. It is worth noting that a perception of this kind could not have been anticipated by naive subjects. Our findings therefore extend those obtained by Kitada et al. (2002) to any motor shape occurring in two-dimensional space.

\section{Common neural processes and networks may mediate kinesthetic illusions and kinesthetic imagery}

The emergence of a single perception combining the features of both the imagined and illusory trajectories constitutes strong evidence in favor of the idea that kinesthetic images of these two kinds involve similar processes and that they may be mediated by a common neural network.

Interestingly, the perceptual integration of two or more kinesthetic sensations has been extensively described in the literature. First, within a single sensory modality, it has been established, for example, that two illusory movements induced in orthogonal directions by applying appropriate vibratory stimuli result in a single perception of a movement with an oblique trajectory when evoked simultaneously (Roll et al., 1996). Second, when different modalities are combined, costimulating the hand palm skin with a dynamic tactile stimulus while a proprioceptive stimulus is being applied to the wrist muscles also gives rise to a single perception showing the spatiotemporal parameters of both stimuli combined (Kavounoudias et al., 2008). Likewise, the "Pinocchio illusion" (Lackner, 1988), a conflicting situation between tactile and proprioceptive feedback, results in a single, although aberrant, sensation of nose lengthening. In any case, two or more kinesthetic sensations cannot coexist in a given body segment, simply because each of our actions is characterized by a single directional goal and velocity at a time. In the present study, the "perceptual conflict" was solved by producing an intermediate kinesthetic perception.

The importance of the sensory component of the motor images generated by imagination was first attested by the fact that a subject can experience the feeling that he is moving his hand in the absence of any proprioceptive feedback (Grush, 2004). In addition, although motor imagery has been assumed to be mediated by similar processes to those involved in motor preparation (Jeannerod, 1994; Jeannerod and Frak, 1999), the various descriptions of kinesthetic motor imagery published so far (Annett, 1995; Magill, 1998) emphasize the fact that "the person actually experiences the sensory sensations that might be expected in that situation" (Mulder, 2007). Motor imagery may therefore involve both the activation of an action plan and a "substitute for the sensory feedback" usually evoked by this action (Annett, 1996). Grush (2004) has suggested the existence of a "body emulator" receiving signals from the motor command and generating kinesthetic experiences during motor imagery. All these findings suggest that the sensory experiences elicited by motor imagery may mimic those that would have been associated with the action if it had been actually executed. In the present case, the occurrence of a fused kinesthetic perception might be attributable to the central processing of both the supposed efferent copy information and the sensory feedback, which raises the question as to what the weighted contribution of each component to kinesthesia may be.

The fact that the motor events associated with imagined or vibration-induced movement sensations may alter both the subject's perceptions and their voluntary drawings of the trajectories perceived needs to be briefly discussed. Changes in cortical and spinal excitability may have occurred, along with an efferent copy generated by the motor command itself. In addition, even a weak muscle activation (involving only one or a few motor units) may induce force feedback messages originating from the Golgi tendon organs (Jami, 1992; Proske, 2005; Gandevia et al., 2006), which, in addition to the efferent copy information, may affect the subject's kinesthetic perceptions. These motor effects may have contributed to the way the perceived trajectories were voluntarily drawn by hand. However, because the drawings were performed after the end of the experimental procedure, these effects can reasonably be assumed to have been negligible. This would not have been the case if the drawings had been produced concomitantly with the perceptual events. In the same way, the possible contribution of variations in motor excitability attributable to changes in the subjects' attentional states can be assumed to have been weak because the subjects were instructed to permanently focus their attention on the treated hand (Rosenkranz and Rothwell, 2006).

That visual and auditory imagery activate the corresponding cortical areas suggests the existence of a high level of correspondence between perception and imagery (Johnson et al., 2002). Likewise, a neural network including specific parietal and motor regions is activated during the perception of vibration-induced illusory movements (Romaiguère et al., 2003; Duclos et al., 2007; Kavounoudias et al., 2008), and this network seems to also play a crucial role in eliciting kinesthetic imagery. Indeed, most neuroimaging studies on motor imagery have shown that practically the same brain network is consistently activated in this context (Decety et al., 1994; Stephan et al., 1995; Lotze et al., 1999; Binkofski et al., 2000; Gerardin et al., 2000), that it is somatotopically organized (Stippich et al., 2002; Ehrsson et al., 2003), and that superior parietal regions are consistently involved (Leonardo et al., 1995; Parsons et al., 1995; Seitz et al., 1997; Wolbers et al., 2003). These findings have been supported by clinical observations showing that the ability to generate motor imagery is altered in patients with parietal lesions (Sirigu et al., 1996). The parietal cortex may therefore contribute crucially to the ability to generate motor images internally or in response to muscle vibration, necessarily in conjunction with the motor cortical network (Naito et al., 2002; Casini et al., 2006).

In conclusion, the present study brings to light the existence of strong analogies between proprioceptive illusions and motor imagery. The fact that these two kinesthetic sensations can be combined into a single percept suggests that the underlying processes and the neural substrates involved are probably very similar. These findings are potentially applicable in the field of sensorimotor rehabilitation.

\section{References}

Albert F, Bergenheim M, Ribot-Ciscar E, Roll JP (2006) The Ia afferent feedback of a given movement evokes the illusion of the same movement when returned to the subject via muscle tendon vibration. Exp Brain Res 172:163-174.

Annett J (1995) Motor imagery: perception or action? Neuropsychologia 33:1395-1417. 
Annett J (1996) On knowing how to do things: a theory of motor imagery. Brain Res Cogn Brain Res 3:65-69.

Batschelet E (1981) Circular statistics in biology. London: Academic.

Benhamou S (2004) How to reliably estimate the tortuosity of an animal's path: straightness, sinuosity, or fractal dimension? J Theor Biol 229:209-220.

Binkofski F, Amunts K, Stephan KM, Posse S, Schormann T, Freund HJ, Zilles K, Seitz RJ (2000) Broca's region subserves imagery of motion: a combined cytoarchitectonic and fMRI study. Hum Brain Mapp 11:273-285.

Calvin-Figuière S, Romaiguère P, Gilhodes JC, Roll JP (1999) Antagonist motor responses correlate with kinesthetic illusions induced by tendon vibration. Exp Brain Res 124:342-350.

Calvin-Figuière S, Romaiguère P, Roll JP (2000) Relations between the directions of vibration-induced kinesthetic illusions and the pattern of activation of antagonist muscles. Brain Res 881:128-138.

Casini L, Romaiguère P, Ducorps A, Schwartz D, Anton JL, Roll JP (2006) Cortical correlates of illusory hand movement perception in humans: a MEG study. Brain Res 1121:200-206.

Collins DF, Refshauge KM, Todd G, Gandevia SC (2005) Cutaneous receptors contribute to kinesthesia at the index finger, elbow, and knee. J Neurophysiol 94:1699-1706.

Decety J, Michel F (1989) Comparative analysis of actual and mental movement times in two graphic tasks. Brain Cogn 11:87-97.

Decety J, Jeannerod M, Germain M, Pastene J (1991) Vegetative response during imagined movement is proportional to mental effort. Behav Brain Res 42:1-5.

Decety J, Jeannerod M, Durozard D, Baverel G (1993) Central activation of autonomic effectors during mental simulation of motor actions in man. J Physiol 461:549-563.

Decety J, Perani D, Jeannerod M, Bettinardi V, Tadary B, Woods R, Mazziotta JC, Fazio F (1994) Mapping motor representations with positron emission tomography. Nature 371:600-602.

Duclos C, Roll R, Kavounoudias A, Roll JP (2007) Cerebral correlates of the "Kohnstamm phenomenon": an fMRI study. Neuroimage 34:774-783.

Ehrsson HH, Geyer S, Naito E (2003) Imagery of voluntary movement of fingers, toes, and tongue activates corresponding body-part-specific motor representations. J Neurophysiol 90:3304-3316.

Gandevia SC, Smith JL, Crawford M, Proske U, Taylor JL (2006) Motor commands contribute to human position sense. J Physiol 571:703-710.

Gerardin E, Sirigu A, Lehéricy S, Poline JB, Gaymard B, Marsault C, Agid Y, Le Bihan D (2000) Partially overlapping neural networks for real and imagined hand movements. Cereb Cortex 10:1093-1104.

Goodwin GM, McCloskey DI, Matthews PB (1972) Proprioceptive illusions induced by muscle vibration: contribution by muscle spindles to perception? Science 175:1382-1384.

Grush R (2004) The emulation theory of representation: motor control, imagery, and perception. Behav Brain Sci 27:377-396.

Hagbarth KE, Eklund G (1966) Motor effects of vibratory stimuli in man. In: Muscular afferents and motor control, Nobel Symposium I (Granit R, ed), pp 177-186. Stockholm: Almqvist and Wiksell.

Jaeger RJ, Agarwal GC, Gottlieb GLJ (1979) Directional errors of movement and their correction in a discrete tracking task. Mot Behav 11:123-133.

Jami L (1992) Golgi tendon organs in mammalian skeletal muscle: functional properties and central actions. Physiol Rev 72:623-666.

Jeannerod M (1994) The representing brain: neural correlates of motor intention and imagery. Behav Brain Sci 17:187-245.

Jeannerod M, Frak V (1999) Mental imaging of motor activity in humans. Curr Opin Neurobiol 9:735-739.

Johnson SH, Rotte M, Grafton ST, Hinrichs H, Gazzaniga MS, Heinze HJ (2002) Selective activation of a parietofrontal circuit during implicitly imagined prehension. Neuroimage 17:1693-1704.

Jones LA (1988) Motor illusions: what do they reveal about proprioception? Psychol Bull 103:72-86.

Kavounoudias A, Roll JP, Anton JL, Nazarian B, Roth M, Roll R (2008) Proprio-tactile integration for kinesthetic perception: an fMRI study. Neuropsychologia 46:567-575.

Kitada R, Naito E, Matsumura M (2002) Perceptual changes in illusory wrist flexion angles resulting from motor imagery of the same wrist movements. Neuroscience 109:701-707.

Kito T, Hashimoto T, Yoneda T, Katamoto S, Naito E (2006) Sensory pro- cessing during kinesthetic aftereffect following illusory hand movement elicited by tendon vibration. Brain Res 1114:75-84.

Lackner JR (1988) Some proprioceptive influences on the perceptual representation of body shape and orientation. Brain 111:281-297.

Leonardo M, Fieldman J, Sadato N, Campbell G, Ibañez V, Cohen L, Deiber MP, Jezzard P, Pons T, Turner R, Le Bihan D, Hallett M (1995) A functional magnetic resonance imaging study of cortical regions associated with motor task execution and motor ideation in humans. Hum Brain Mapp 3:83-92.

Lotze M, Halsband U (2006) Motor imagery. J Physiol Paris 99:386-395.

Lotze M, Montoya P, Erb M, Hülsmann E, Flor H, Klose U, Birbaumer N, Grodd W (1999) Activation of cortical and cerebellar motor areas during executed and imagined hand movements: an fMRI study. J Cogn Neurosci 11:491-501.

Magill RA (1998) Motor learning: concepts and applications. New York: McGraw Hill.

McCloskey DI (1981) Corollary discharges: motor commands and perception. In: Handbook of physiology. The nervous system, motor control (Brooks VB, ed), pp 1415-1447. Bethesda, MD: American Physiological Society.

Mercier C, Aballea A, Vargas CD, Paillard J, Sirigu A (2008) Vision without proprioception modulates cortico-spinal excitability during hand motor imagery. Cereb Cortex 18:272-277.

Mulder T (2007) Motor imagery and action observation: cognitive tools for rehabilitation. J Neural Transm 114:1265-1278.

Naito E, Kochiyama T, Kitada R, Nakamura S, Matsumura M, Yonekura Y, Sadato N (2002) Internally simulated movement sensations during motor imagery activate cortical motor areas and the cerebellum. J Neurosci 22:3683-3691.

Parsons LM (1994) Temporal and kinematic properties of motor behavior reflected in mentally simulated action. J Exp Psychol Hum Percept Perform 20:709-730.

Parsons LM, Fox PT, Downs JH, Glass T, Hirsch TB, Martin CC, Jerabek PA, Lancaster JL (1995) Use of implicit motor imagery for visual shape discrimination as revealed by PET. Nature 375:54-58.

Porro CA, Francescato MP, Cettolo V, Diamond ME, Baraldi P, Zuiani C, Bazzocchi M, di Prampero PE (1996) Primary motor and sensory cortex activation during motor performance and motor imagery: a functional magnetic resonance imaging study. J Neurosci 16:7688-7698.

Proske U (2005) What is the role of muscle receptors in proprioception? Muscle Nerve 31:780-787.

Roll JP, Vedel JP (1982) Kinesthetic role of muscle afferents in man, studied by tendon vibration and microneurography. Exp Brain Res 47:177-190.

Roll JP, Gilhodes JC, Tardy-Gervet MF (1980) Effects of vision on tonic vibration response of a muscle or its antagonists in normal man. Experientia 36:70-72.

Roll JP, Gilhodes JC, Roll R (1994) Kinesthetic illusions as tools in understanding motor imagery. Behav Brain Sci 17:220-221.

Roll JP, Gilhodes JC, Roll R, Harlay F (1996) Are proprioceptive sensory inputs combined into a "gestalt"? Vibration-induced virtual hand drawing or visual target motion. In: Attention and performance (Inui T, McClelland JL, eds), Vol 12, pp 291-314. London: MIT.

Roll R, Kavounoudias A, Roll JP (2002) Cutaneous afferents from human plantar sole contribute to body posture awareness. Neuroreport 13:1957-1961.

Roll JP, Albert F, Thyrion C, Ribot-Ciscar E, Bergenheim M, Mattei B (2009) Inducing any virtual two-dimensional movement in humans by applying muscle tendon vibration. J Neurophysiol 101:816-823.

Romaiguère P, Anton JL, Roth M, Casini L, Roll JP (2003) Motor and parietal cortical areas both underlie kinaesthesia. Brain Res Cogn Brain Res 16:74-82.

Rosenkranz K, Rothwell JC (2004) The effect of sensory input and attention on the sensorimotor organization of the hand area of the human motor cortex. J Physiol 561:307-320.

Rosenkranz K, Rothwell JC (2006) Spatial attention affects sensorimotor reorganization in human motor cortex. Exp Brain Res 170:97-108.

Seitz RJ, Canavan AG, Yágüez L, Herzog H, Tellmann L, Knorr U, Huang Y, Hömberg V (1997) Representations of graphomotor trajectories in the human parietal cortex: evidence for controlled processing and automatic performance. Eur J Neurosci 9:378-389.

Sirigu A, Duhamel JR, Cohen L, Pillon B, Dubois B, Agid Y (1996) The 
mental representation of hand movements after parietal cortex damage. Science 273:1564-1568.

Smith JL, Crawford M, Proske U, Taylor JL, Gandevia SC (2009) Signals of motor command bias joint position sense in the presence of feedback from proprioceptors. J Appl Physiol 106:950-958.

Stephan KM, Fink GR, Passingham RE, Silbersweig D, Ceballos-Baumann AO, Frith CD, Frackowiak RSJ (1995) Functional anatomy of the mental representation of upper extremity movements in healthy subjects. J Neurophysiol 73:373-386.

Stippich C, Ochmann H, Sartor K (2002) Somatotopic mapping of the human primary sensorimotor cortex during motor imagery and motor execution by functional magnetic resonance imaging. Neurosci Lett 331:50-54.

Tardy-Gervet MF, Gilhodes JC, Roll JP (1982) Demonstration of an illusory limb movement and associated motor activities induced by a moving visual stimulus in man. A descriptive study. Neurosci Lett 28:187-192.

Teuber HL (1972) Perception et mouvements, aspects neurophysiologiques et psychophysiologiques. In: Neuropsychologie de la perception visuelle (Hecaen H, ed), pp 187-221. Paris: Masson.

Von Holst E (1954) Relations between the central nervous system and the peripheral organs. Br J Anim Behav 2:89-94.

Wolbers T, Weiller C, Büchel C (2003) Contralateral coding of imagined body parts in the superior parietal lobe. Cereb Cortex 13:392-399.

Wuyam B, Moosavi SH, Decety J, Adams L, Lansing RW, Guz A (1995) Imagination of dynamic exercise produced ventilatory responses which were more apparent in competitive sportsmen. J Physiol 48:713-724. 